\title{
Unusual presentation of aortopulmonary septal defect and aortic arch interruption
}

\section{Presentación inusual de ventana aortopulmonar e interrupción aórtica}

\author{
Rocío A. Peña-Juárez ${ }^{*}$, Miguel A. Medina-Andrade ${ }^{1}$, Carlos A. Corona-Villalobos², and
} Juan P. Abarca-De la Paz ${ }^{1}$

${ }^{1}$ Department of Pediatric Cardiology, Hospital General de Occidente, Zapopan, Jalisco; ${ }^{2}$ Department of Pediatric Cardiology, Instituto Nacional de Pediatría, Mexico City, Mexico

Aortopulmonary septal is a rare anomaly that accounts for $0.2-0.6 \%$ of congenital heart diseases; it involves communication between the ascending aorta and the pulmonary trunk (PT) in the presence of both sigmoid valve planes. The most widely used classification is that by Mori; in type II (distal), the defect is spiral-shaped and affects the trunk and right branch origin ${ }^{1}$. Around half the patients can exhibit associated alterations: aortic arch interruption accounts for up to $20 \%$, especially type A (interruption between the left subclavian artery origin and the descending aorta) ${ }^{2}$.

We present the case of a newborn with a type II aortopulmonary septal defect with right pulmonary artery abnormal origin, which emerged from the posterior portion of the ascending aorta and gave rise to a type $A$ aortic arch interruption. Heart disease was suspected during physical examination due to the presence of hemodynamic instability within the first hours of life and decreased pulses and blood pressure in lower versus superior limbs. Images obtained by 64-slice multidetector computed tomography, performed with a

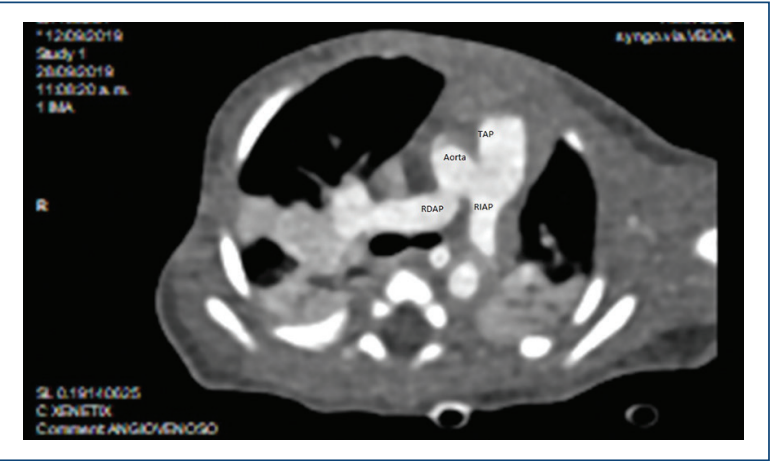

Figure 1. Aortopulmonary septal defect, communication between the aorta and pulmonary trunk (PT); the right pulmonary artery (RPA) originates from the aorta without anomalies at the origin of the left pulmonary artery (LPA).

low-dose protocol $(80 \mathrm{kV}, 50 \mathrm{~mA})$, outlined a type II aortopulmonary septal defect (Fig. 1), showing communication between the PT and the ascending aorta. The right pulmonary artery originated in the posterior 


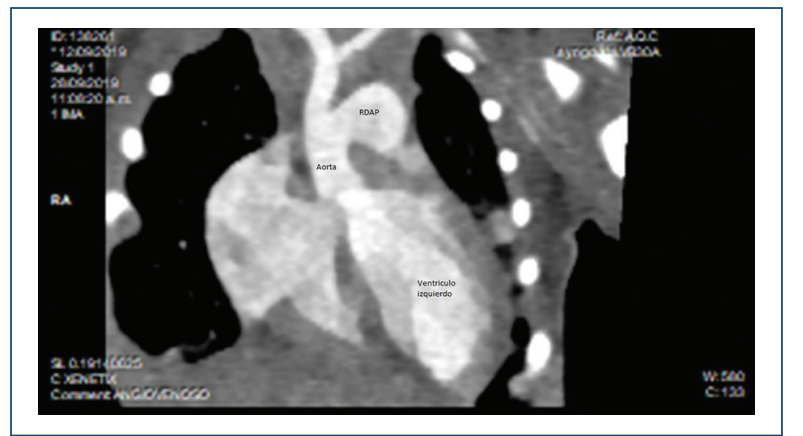

Figure 2. The aorta is observed to emerge from the left ventricle, with right pulmonary artery (RPA) originating from the posterior aspect of the ascending aorta.

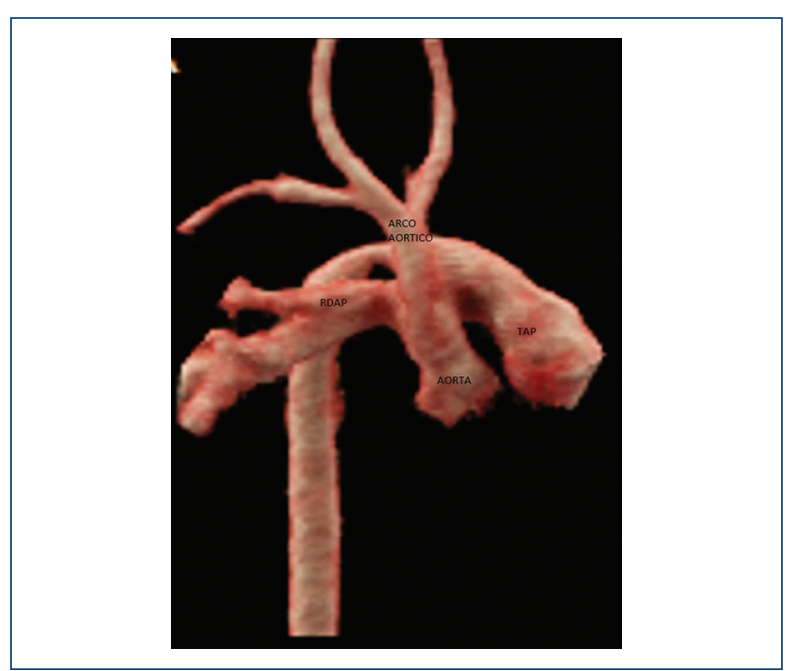

Figure 3. Right pulmonary artery (RPA) abnormal origin and type-A aortic arch interruption. aspect of the ascending aorta (Figs. 1 and 2), with a type A aortic arc interruption being also identified (Fig. 3).

\section{Funding}

None.

\section{Conflicts of interest}

The authors declare that they have no conflicts of interest.

\section{Ethical disclosures}

Protection of human and animal subjects. The authors declare that no experiments were performed on humans or animals for this research.

Confidentiality of data. The authors declare that they have followed the protocols of their work center on the publication of patient data.

Right to privacy and informed consent. The authors have obtained informed consent from the patients or subjects referred to in the article. This document is in the possession of the corresponding author.

\section{References}

1. Mori K, Ando M, Takao A, Ishikawa S, Imai Y. Distal type of aortopulmonary window. Report 4 cases. Br Heart J. 1978;40:681-9.

2. Kutsche LM, Van Mierop LHS. Anatomy and pathogenesis of aortopulmonary septal defect. Am J Cardiol. 1987:59:443-7. 